\title{
TUNGURAHUA Y SU APORTE AL COMERCIO EXTERIOR
}

\author{
ANA MARÍA SÁNCHEZ TOBAR 1
}

\section{EXPORTACIONES DE}

\section{LA PROVINCIA}

Pag 4-6

\section{Resumen}

A partir de información entregada por la Cámara de Industrias de Tungurahua se realiza un análisis sobre las exportaciones efectuadas por la provincia de Tungurahua durante el periodo 2014 a 2016 en valores FOB y en dos aspectos: principales países de destino y sub partidas arancelarias de mayor peso, con esta información se obtiene el número de exportadores por provincia, cuántos de ellos pertenecen al grupo de pequeñas y medianas industrias, y, su ubicación geográfica por cantón. Este artículo evidencia el aporte que realiza Tungurahua al comercio exterior del Ecuador con sus 32 empresas exportadoras registradas en la Federación de Exportadores Ecuatorianos - FEDEXPO.

\section{Palabras clave:}

Exportaciones, países de destino, sub partidas arancelarias

\section{Abstract}

Starting with information from the Industrial Chamber of Tungurahua analysis of the exports from Tungurahua during the period 2014 - 2016, expressed in FOB values, and with a focus on two aspects: main export destination countries and sub-tariffs of greatest weight. With this information it can be established, how many exporters per province, how many of them belong to the small and medium industries and their geographic location by Canton. This paper evidences the important input that Tungurahua gives to Ecuador's foreign trade with its 32 exporting companies registered at the Federation of Ecuadorian Exporters - FEDEXPO

\section{Keywords:}

Exports, export destination countries, tariffs

\section{Introducción}

El Libro V del Código Orgánico de la Producción, Comercio e Inversiones $\mathrm{COPCl}$, define a la exportación como "el régimen aduanero que permite la salida definitiva de mercancías en libre circulación, fuera del territorio aduanero ecuatoriano o a una Zona Especial de Desarrollo Económico, con sujeción a las disposiciones establecidas en la normativa legal vigente"2.

La página web de la Federación Ecuatoriana de Exportadores FEDEXPO, registra en su directorio de empresas 2.130 exportadores a nivel nacional, la provincia de Pichincha ocupa el primer puesto con 825 empresas, es decir, el 39\%, el segundo puesto ocupa Guayas con 700 empresas, seguido de Manabí, El Oro, Azuay, Cotopaxi, Imbabura, Santo Domingo de los Tsáchilas y Los Ríos hasta llegar en el puesto diez la provincia de Tungurahua con 32 empresas que representan el $2 \%$ del total nacional.

${ }^{1}$ Economista - Especialista de Investigación y Desarrollo Observatorio Económico y Social de Tungurahua - OBEST - UTA

${ }^{2}$ Guía del Exportador - Ministerio de Comercio Exterior, Pág. 2

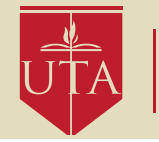

UNIVERSIDAD TÉCNICA DE AMBATO

\section{Figura 1. Porcentaje de exportadores por provincia Ecuador 2013}

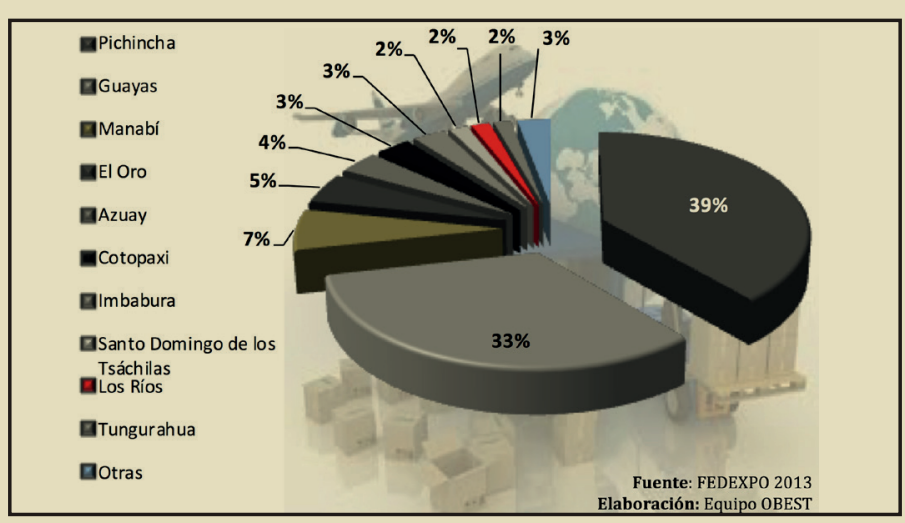

Según definición del Servicio de Rentas Internas - SRI, se conoce como PYME "al conjunto de pequeñas y medianas empresas que de acuerdo a su volumen de ventas, capital social, cantidad de trabajadores, y su nivel de producción o activos presentan características propias de este tipo de entidades económicas". Las PYME pueden desarrollar diferentes actividades económicas como comercio, agricultura, pesca, silvicultura, industria manufacturera, construcción, transporte, almacenamiento y comunicaciones, bienes inmuebles, y servicios empresariales, sociales y/o personales. Las PYME tienen una gran importancia en el aporte económico y generación de empleo que brindan en el país y más aún si su actividad se complementa con exportaciones.

\section{Contenido}

De 2.130 empresas que constan en el directorio de FEDEXPO como exportadoras, el $60 \%$ están registradas como PYME, en Tungurahua el porcentaje es de 65\%.

\section{Figura 2. Porcentaje de exportadores por cantón Tungurahua 2013}

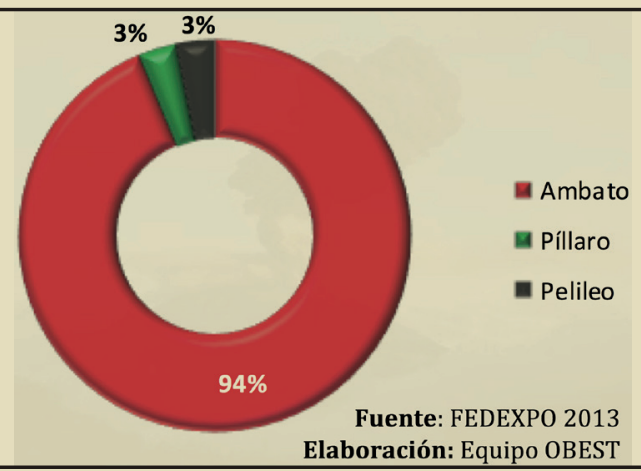

Siendo Ambato la capital de Tungurahua cuenta con el 94\% de exportadores registrados, sin embargo, se observa también una empresa en el cantón Píllaro y otra en Pelileo. El número mayoritario de empresas exportadoras en Ecuador se encuentran localizadas en los cantones de las diferentes provincias, a pesar de ello, existen dos provincias en donde no ocurre el mismo fenómeno, Los Ríos, donde el $50 \%$ de industrias se localizan en Quevedo e Imbabura que alberga el $70 \%$ de empresas exportadoras en Otavalo. 
Tabla 1. Porcentaje de exportadoras en capital de provincia Ecuador 2013

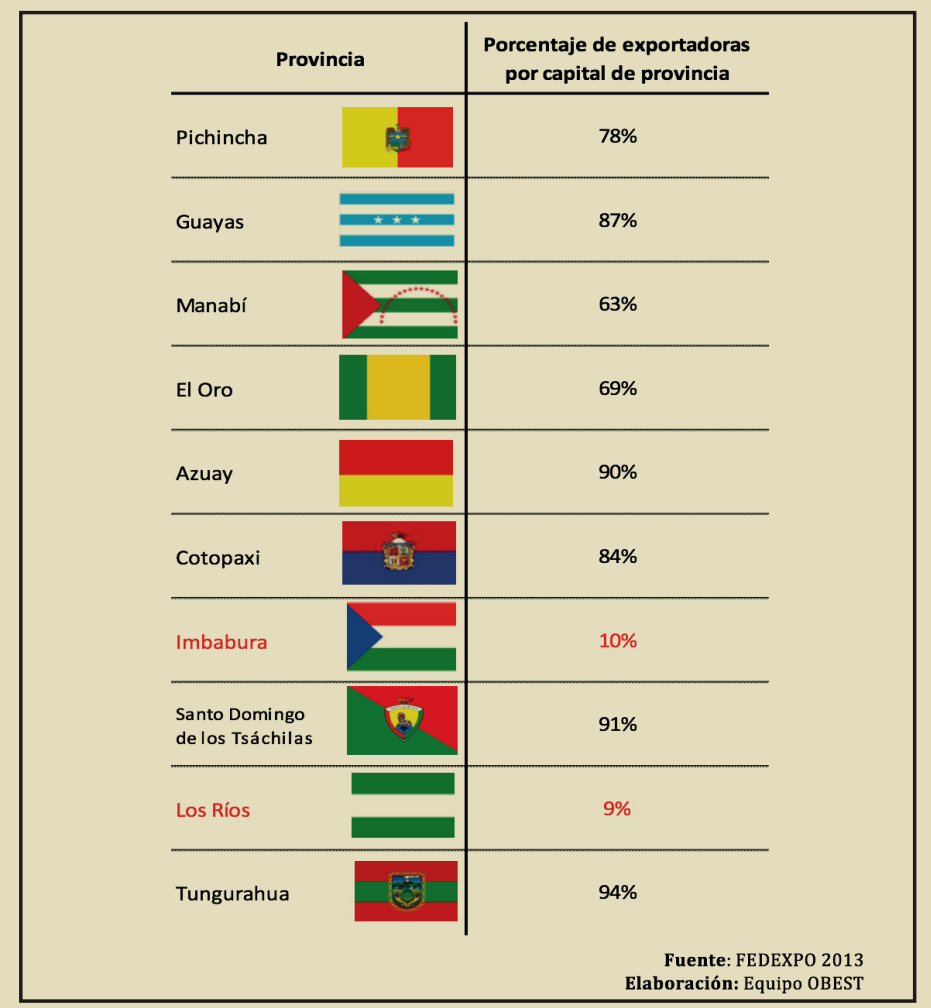

La Cámara de Industrias de Tungurahua - CIT cuenta con 64 socios registrados que trabajan en diferentes ramas industriales, entre ellas, rama alimenticia, caucho y cuero, curtiembres, eléctrica y energética, metalmecánica, textil y varias. A partir de información proporcionada por la CIT se presenta el siguiente análisis sobre exportaciones de la provincia de Tungurahua desde el año 2014 al 2016.

El 80\% de las exportaciones realizadas por empresas tungurahuenses se concentra en tres países de destino, Colombia con el $63 \%$, Perú 14\% y Venezuela 9\%, el restante 13\% se destina a otros países como Costa Rica, Hong Kong, Bolivia, México, Chile, República Dominicana, Guatemala, Estados Unidos, Panamá, Uruguay, el Salvador, China, España y Vietnam. En la Figura N. 3 se puede observar las exportaciones de Tungurahua en valores FOB, siendo Colombia el principal destino de los productos, se muestra un crecimiento de 57\% del total exportado en el año 2015 con relación a 2014, hasta junio de 2016 el valor exportado a Colombia ha alcanzado el 38\% del total de 2015.

\section{Figura 3. Exportaciones Tungurahua por país de destino (valores FOB) $2014-2016$}

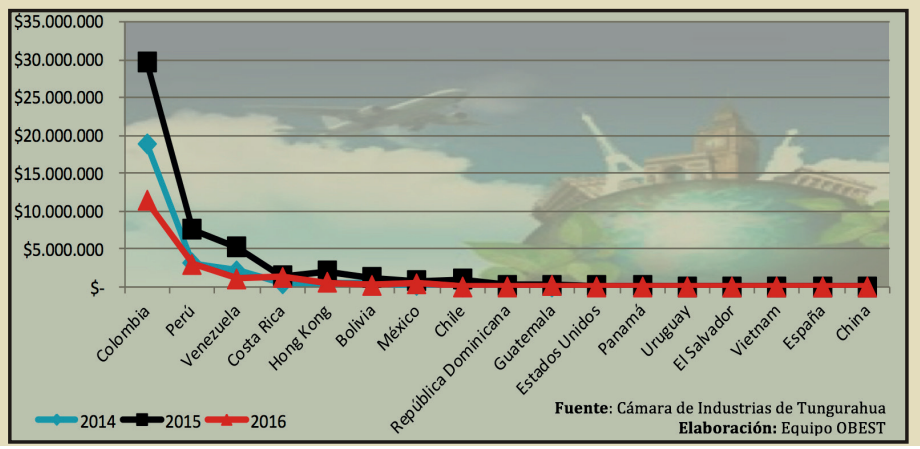

Tabla 2. Exportaciones Tungurahua valores $\mathrm{FOB}$ $2014-2016$

\begin{tabular}{|c|c|c|c|c|c|c|}
\hline Mes & & 2014 & & 2015 & & 2016 \\
\hline Enero & $\$$ & - & $\$$ & $4.385 .621,19$ & $\$$ & $4.126 .775,37$ \\
\hline Febrero & $\$$ & - & $\$$ & $3.853 .885,75$ & $\$$ & $3.526 .722,34$ \\
\hline Marzo & $\$$ & - & $\$$ & $5.010 .944,31$ & $\$$ & $3.224 .927,29$ \\
\hline Abril & $\$$ & - & $\$$ & $2.919 .488,27$ & $\$$ & $3.244 .124,24$ \\
\hline Mayo & $\$$ & - & $\$$ & $4.657 .678,07$ & $\$$ & $2.614 .245,82$ \\
\hline Junio & $\$$ & - & $\$$ & $3.080 .595,96$ & $\$$ & $1.847 .182,11$ \\
\hline Julio & $\$$ & & $\$$ & $4.342 .576,36$ & $\$$ & 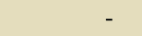 \\
\hline Agosto & $\$$ & $4.496 .877,60$ & $\$$ & $4.497 .548,66$ & $\$$ & - \\
\hline Septiembre & $\$$ & 4.627.441,81 & $\$$ & $4.610 .034,44$ & $\$$ & - \\
\hline Octubre & $\$$ & $6.264 .949,48$ & $\$$ & $4.489 .299,74$ & $\$$ & - \\
\hline Noviembre & $\$$ & 5.388.334,95 & $\$$ & $5.411 .451,18$ & $\$$ & - \\
\hline Diciembre & $\$$ & $5.793 .652,86$ & $\$$ & $2.828 .335,20$ & $\$$ & - \\
\hline Total general & $\$$ & $26.571 .256,70$ & $\$$ & $50.087 .459,13$ & $\$$ & 18.583.977,17 \\
\hline \multicolumn{7}{|c|}{$\begin{array}{r}\text { Fuente: Cámara de Industrias de Tungurahua } \\
\text { Elaboración: Equipo OBEST }\end{array}$} \\
\hline
\end{tabular}

Los meses en los que se presenta mayor volumen de exportaciones en el año 2015 (medido en valores FOB) desde Tungurahua son noviembre y marzo. Los meses de menor volumen en envíos fueron abril y diciembre, mensualmente el valor FOB promedio de envíos a diferentes países en 2014 fue de \$5’314.251 dólares, 27\% más del mensual promedio de 2015 en donde el promedio fue de \$4'173.954,93 dólares. Hasta junio de 2016 el valor FOB promedio mensual registrado fue de \$3’097.329 dólares, cifra similar al promedio del primer semestre de 2015.

\section{Figura 4. Exportaciones promedio mensual Tungurahua (valores FOB) 2015}

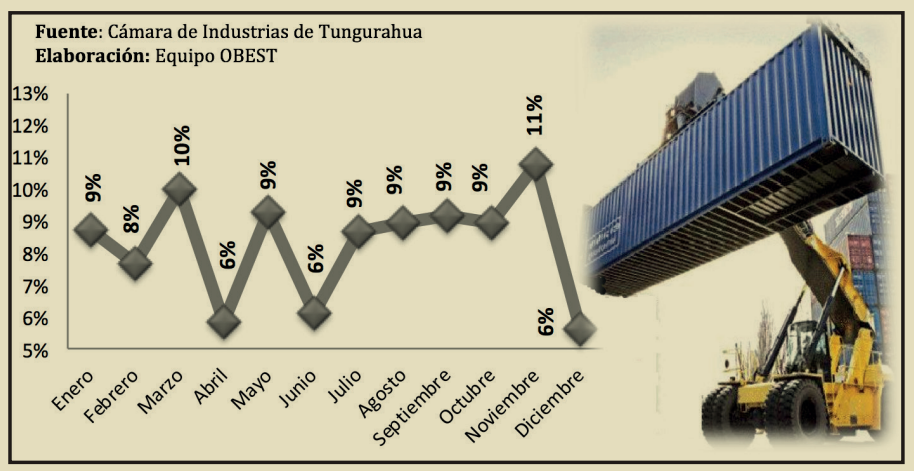

Las principales sub partidas de exportaciones registradas en las exportaciones de Tungurahua de los años 2014, 2015 y 2016 se encuentran dentro de las siguientes sub partidas generales:

-Alforfón, mijo y alpiste; los demás cereales;

-Melaza procedente de la extracción o del refinado del azúcar;

-Alcohol etílico sin desnaturalizar con grado alcohólico volumétrico superior o igual al 80 \% vol.; alcohol etílico y aguardiente desnaturalizados, de cualquier graduación;

- Gelatinas (aunque se presenten en hojas cuadradas o rectangulares, incluso trabajadas en la superficie o coloreadas) y sus derivados; ictiocola; las demás colas de origen animal (excepto las colas de caseína de la partida 3501);

-Polímeros de cloruro de vinilo o de otras olefinas halogenadas, en formas primarias;

- Cueros preparados después del curtido o del secado y cueros y pieles apergaminados, de bovino (incluido el búfalo) o de equino, depilados, incluso divididos (excepto los de la partida 4114);

- Madera contrachapada, madera chapada y madera estratificada similar; 
- Libros registro, libros de contabilidad, talonarios (de notas, pedidos o recibos), agendas, bloques, memorandos, bloques de papel de cartas y artículos similares, cuadernos, carpetas de mesa, clasificadores, encuadernaciones (de hojas móviles u otras);

- Tejidos de algodón con un contenido de algodón superior o igual al $85 \%$ en peso, de peso inferior o igual a $200 \mathrm{~g} / \mathrm{m}^{2}$;

- Tejidos de algodón con un contenido de algodón superior o igual al $85 \%$ en peso, de peso superior a $200 \mathrm{~g} / \mathrm{m}^{2}$;

- Cintas (excepto los artículos de la partida 5807); cintas sin trama, de hilados o fibras paralelizados y aglutinados;

- Ropa de cama, de mesa, de tocador o cocina;

- Los demás artículos confeccionados, incluidos los patrones para prendas de vestir;

-Calzado impermeable con suela y parte superior de caucho o plástico, cuya parte superior no se haya unido a la suela por costura o por medio de remaches, clavos, tornillos, espigas o dispositivos similares, ni se haya formado con diferentes partes unidas;

- Los demás calzados con suela y parte superior de caucho o plástico;
- Calzado con suela de caucho, plástico, cuero natural o regenerado y parte superior de cuero natural;

- Calzado con suela de caucho, plástico, cuero natural o regenerado y parte superior de materia textil;

-Partes de calzado, incluidas las partes superiores fijadas a las palmillas distintas de la suela; plantillas, taloneras y artículos similares, amovibles; polainas y artículos similares, y sus partes;

- Hilados de algodón (excepto el hilo de coser) con un contenido de algodón, superior o igual al 85 \% en peso, sin acondicionar para la venta al por menor;

- Vidrio de seguridad constituido por vidrio templado o contrachapado; y,

- Transformadores eléctricos, convertidores eléctricos estáticos (por ejemplo: rectificadores) y bobinas de reactancia (autoinducción).

La industria de cuero y calzado a nivel de Tungurahua genera un importante aporte, encontrándose dentro de las cuatro provincias con mayor desarrollo de la industria según PROECUADOR. A continuación en la Tabla Nro. 3 se detallan las principales sub partidas arancelarias de exportación según valor FOB en los años 2014, 2015 y 2016 :

\section{Tabla 3. Principales sub partidas de exportación de Tungurahua valores FOB \\ 2014, 2015 y 2016}

\begin{tabular}{|c|c|c|c|c|c|}
\hline Subpartida & Valor FOB & Subpartida & Valor FOB & Subpartida & Valor FOB \\
\hline $\begin{array}{l}\text { Los demás calzados, que } \\
\text { cubran el tobillo sin cubrir } \\
\text { la rodilla }\end{array}$ & $\$ 25.730 .233$ & $\begin{array}{l}\text { Calzado con suela de } \\
\text { caucho, plástico, cuero } \\
\text { natural y parte superior de } \\
\text { materia textil }\end{array}$ & $\$ 8.502 .828$ & $\begin{array}{l}\text { Demás cueros y pieles de } \\
\text { bovino y de equino apergaminados o } \\
\text { preparados después del } \\
\text { curtido, excepto con la } \\
\text { flor, incluso dividido, } \\
\text { y de las partidas } \\
41.08 \text { ó } 41.09\end{array}$ & $\$ \quad 4.646 .742$ \\
\hline $\begin{array}{l}\text { Gelatinas y } \\
\text { sus derivados }\end{array}$ & $\$ 19.260 .830$ & $\begin{array}{l}\text { Partes superiores de } \\
\text { calzado y sus partes } \\
\text { (excepto los } \\
\text { contrafuertes y } \\
\text { punteras duras) }\end{array}$ & $\$ 7.455 .123$ & $\begin{array}{l}\text { Los demás, con plantilla de } \\
\text { longitud, inferior a } 24 \mathrm{~cm}\end{array}$ & $\$ \quad 4.543 .880$ \\
\hline Plastificados & $\$ 10.125 .358$ & $\begin{array}{l}\text { Vidrio de } \\
\text { seguridad }\end{array}$ & $\$ 6.406 .643$ & $\begin{array}{l}\text { Calzado de tenis, } \\
\text { baloncesto, gimnasia, } \\
\text { entrenamiento y } \\
\text { calzados similares }\end{array}$ & $\$ \quad 2.948 .456$ \\
\hline
\end{tabular}

Fuente: Cámara de Industrias de Tungurahua Elaboración: Equipo OBEST

En valores menos representativos se exporta también transformadores, pieles, cuadernos, quinoa, alcohol etílico, textiles, melaza de caña, entre otros.

\section{REFERENCIAS}

-Ministerio de Comercio Exterior del Ecuador. (2014). Guía del Exportador. Disponible en: http://spotidoc.com/doc/1433631/gu\%С3\%АЗ\% C2\%ADa-del-exportador

-Federación Ecuatoriana de Exportadores - FEDEXPO. (2013). Directorio de Empresas Exportadoras. Disponible en: $h t t p: / / w w w . f e d e x-$ por.com/directorio/empresas-exportadoras

-Servicio de Rentas Internas - SRI. ¿Qué son las PYMES?. Disponible en: http://www.sri.gob.ec/web/guest/pymes 